\title{
Chapter 6 \\ Application of SARS-CoV-2 Serology Testing: A Case Study
}

\author{
Masaki Yamamoto, Yasufumi Matsumura, and Miki Nagao
}

\begin{abstract}
Coronavirus disease 2019 (COVID-19) caused by the new coronavirus, severe acute respiratory syndrome coronavirus 2 (SARS-CoV-2) emerged in early December 2019, in Wuhan city, the capital city of Hubei Province. This virus spread easily and rapidly worldwide. A lot of serology testing for COVID-19 are now available, and very powerful tools to understand the immune response to SARSCoV-2, vaccine efficacy, hard immunity among communities, and so on. However, there remains several uncertainties about serology testing, compared to the direct virus detection test, such as reverse transcription polymerase chain reaction (RTPCR) and antigen test. In this chapter, we introduce the current understanding of serological testing for COVID-19 to clarify how to use and select during the COVID19 pandemic.
\end{abstract}

Keywords Sars-Cov-2 $\cdot$ Serology testing $\cdot$ COVID-19 pandemic

\section{What is SARS-CoV-2 Serology Testing?}

Diagnostic modalities for COVID-19 infection used in clinical settings include the nucleic acid amplification test (NAAT), antigen testing, and serology testing. Serology testing, also known as antibody testing, is usually conducted after complete recovery from COVID-19. While serological immunoassays are now widely available from many diagnostic manufacturers globally, there is significant debate regarding their clinical utility, as well as the appropriate clinical and analytical performance characteristics for routine applications during the current pandemic.

To date, there have been few guidelines or statements regarding serological testing. Serological testing has been used to confirm the prevalence of COVID-19 infection in certain cohorts, such as communities or healthcare professionals.

M. Yamamoto $(\varangle) \cdot$ Y. Matsumura · M. Nagao

Department of Clinical Laboratory Medicine, Kyoto University Graduate School of Medicine,

Kyoto, Japan

e-mail: masakiy@kuhp.kyoto-u.ac.jp

M. Yano et al. (eds.), Socio-Life Science and the COVID-19 Outbreak, Economics, Law, and Institutions in Asia Pacific, https://doi.org/10.1007/978-981-16-5727-6_6 
In addition, serological testing can be used for the following purposes:, ${ }^{12}$

- to quantitatively evaluate the antibody response in COVID-19 patients;

- to assist in identifying potential convalescent plasma donors;

- to assist in identifying immunity and evaluating the antibody response to vaccines;

- to assist in monitoring the progression of herd immunity.

As such, serological testing offers marked potential to understand COVID-19. However, just like for NAAT, each serological test exhibits different sensitivity and specificity among each testing method, and the antigen used for detection in each assay differs, which will consequently affect the sensitivity and specificity as well.

In this chapter, we briefly describe the type and selection of serological testing and a case study involving a cohort of essential workers in Kyoto city.

\section{Antibody Testing for COVID-19: Types and Selection}

The diagnosis of COVID-19 is mainly based on viral nucleic acid detection, such as by the reverse transcription-polymerase chain reaction (RT-PCR). Although RT-PCR assays generally have high sensitivity (71-98\%), false-negative results can occur due to inappropriate sample collection. ${ }^{3}$ Furthermore, more than $80 \%$ of people infected with COVID-19, especially young people, are known to be asymptomatic or have mild disease. It is difficult to diagnose such people with COVID-19. Therefore, it is problematic to clarify the actual prevalence of COVID-19 using direct detection methods.

If pathogenic microorganisms, including SARS-CoV-2, infect humans, immune responses occur in the body. In this process, the production of specific antibodies (immunoglobulins) by the host's plasma B cells on encountering the pathogens comprises one of the most important immune responses. These responses can occur even if the person has no obvious symptoms of infection (for example, fever, cough, and shortness of breath). Antibody tests, also known as serology tests, detect the host's immune response to the infection rather than detecting the virus itself. Antibody tests are used to detect specific antibodies in human blood and are considered as important methods in epidemiological research and to monitor seropositivity. Moreover, a serology test can provide information on the immune status after vaccination, and could help determine appropriate donors of convalescent plasma.

For example, in the guidelines of the Centers for Disease Control and Prevention (CDC) in America, the antibody test has been designed and validated for surveillance and research purposes. Its purpose is to estimate the percentage of the population

\footnotetext{
${ }^{1}$ Bohn et al. (2020).

${ }^{2}$ Interim Guidelines for COVID-19 Antibody Testing.

${ }^{3}$ The Infectious Diseases Society of America Guidelines on the Diagnosis of COVID-19: Molecular Diagnostic Testing.
} 
previously infected with SARS-CoV-2, which is necessary to guide the response to the pandemic and protect the public's health. ${ }^{4}$

\subsection{CDC Guidelines and EUA of Antibody Testing by FDA}

The CDC summarized antibody tests for COVID-19 in their "Interim Guidelines for COVID-19 Antibody Testing", as follows ${ }^{5}$ :

- Several serologic assays for SARS-CoV-2 have received Emergency Use Authorization (EUA) by the US Food and Drug Administration (FDA), which has independently reviewed their performance.

- Currently, there is no known advantage of assays testing for $\operatorname{IgG}, \operatorname{IgM}, \operatorname{IgG}$, or total antibody.

- It is important to minimize false-positive test results by choosing an assay with high specificity and by testing populations and individuals with an elevated likelihood of previous exposure to SARS-CoV-2. Alternatively, an orthogonal testing algorithm (that is, employing two independent tests in sequence when the first test yields a positive result) can be used when the expected positive predictive value of a single test is low.

- Antibodies most commonly become detectable 1-3 weeks after symptom onset, at which time evidence suggests that infectiousness markedly decreases and some degree of immunity from future infection has developed. However, additional data are needed before revising public health recommendations based on serologic test results, including decisions on discontinuing physical distancing and using personal protective equipment.

At the end of December 2020, many antibody tests for COVID-19 had been made available and approved by the FDA under EUA. ${ }^{6}$ (Table 1) These tests show high sensitivity and specificity. However, the sensitivity of LFAs is relatively low compared with other laboratory assays, such as ELISA and other high-throughput assays. According to the "Interim Guidelines for COVID-19 Antibody Testing" by the CDC, antibody tests with sufficiently high specificity (for example, > 99.5\%) are required to ensure a high positive predictive value (for example, 95\%). Therefore, ELISA and other high-throughput assays are more appropriate than LFAs for seroprevalence surveillance.

\footnotetext{
${ }^{4}$ Interim Guidelines for COVID-19 Antibody Testing.

${ }^{5}$ Interim Guidelines for COVID-19 Antibody Testing.

${ }^{6}$ EUA Authorized Serology Test Performance.
} 


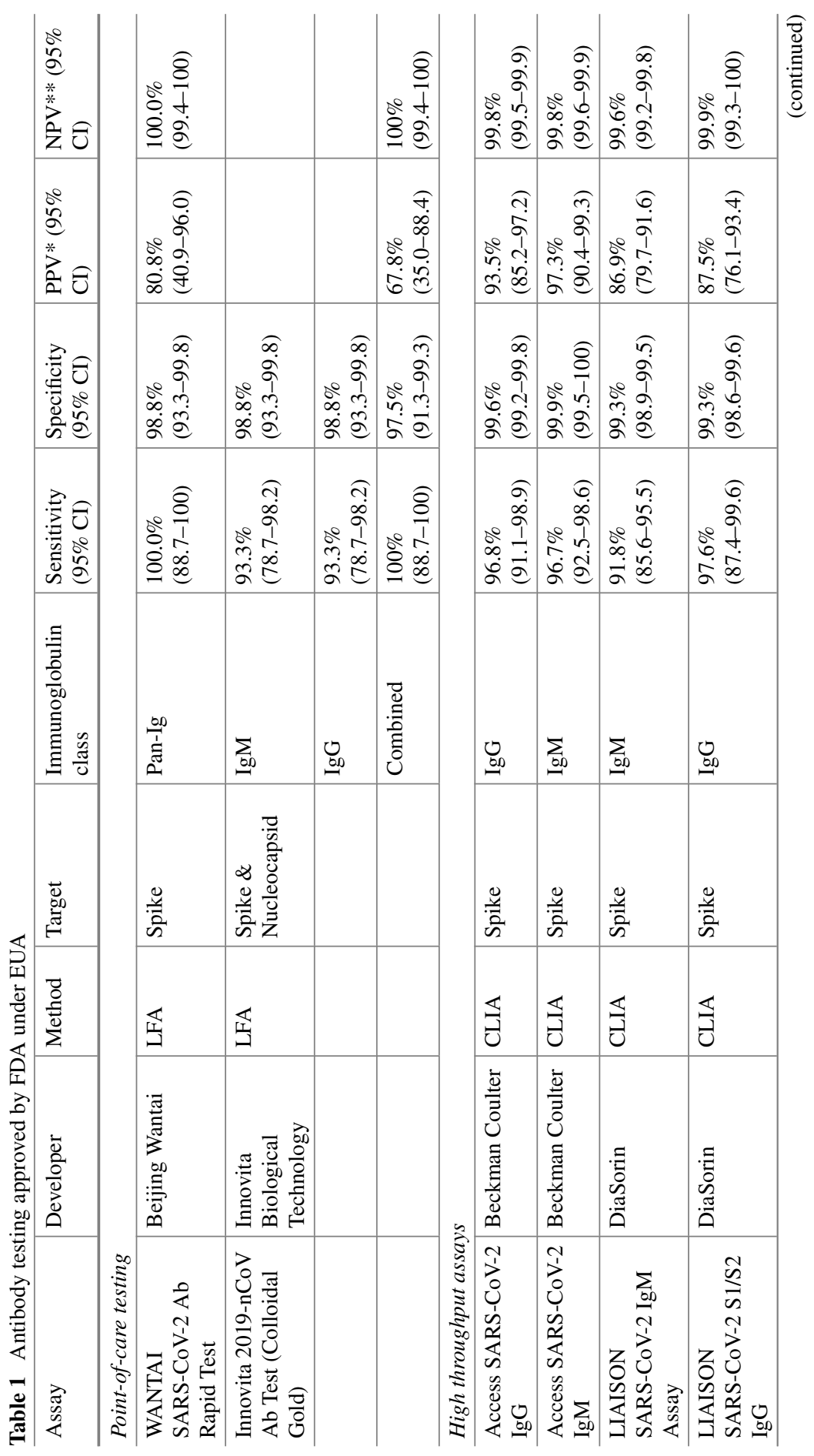




\begin{tabular}{|c|c|c|c|c|c|c|c|c|}
\hline 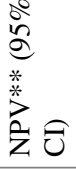 & 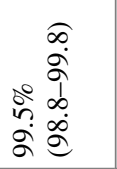 & 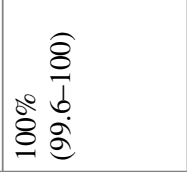 & 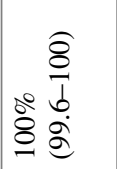 & 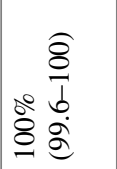 & 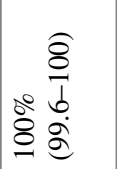 & 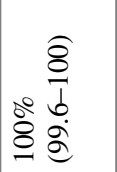 & 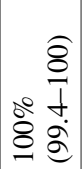 & 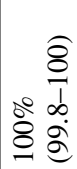 \\
\hline 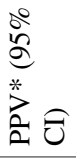 & 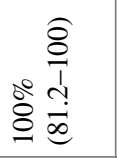 & 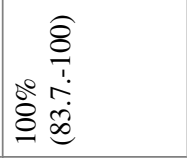 & 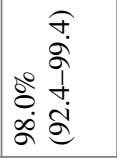 & 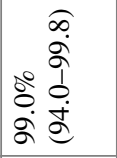 & 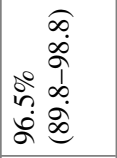 & 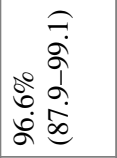 & 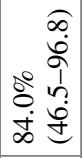 & 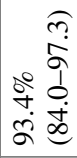 \\
\hline 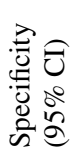 & $\begin{array}{c}\hat{8} \\
\frac{1}{1} \\
8 \\
8\end{array}$ & 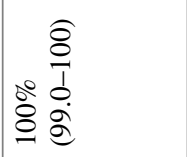 & 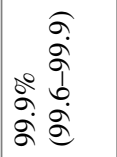 & 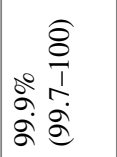 & 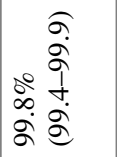 & 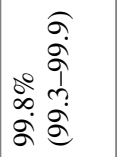 & 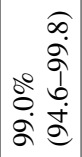 & 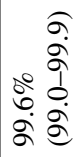 \\
\hline 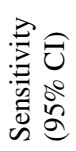 & 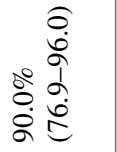 & 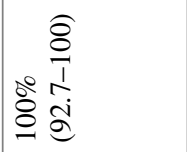 & 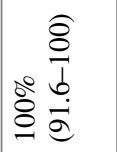 & 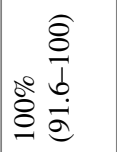 & 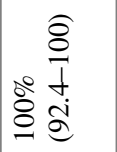 & 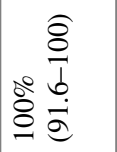 & 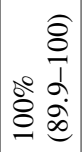 & 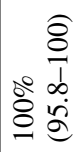 \\
\hline 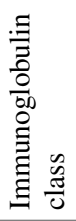 & $\begin{array}{l}\unlhd \\
\Xi\end{array}$ & 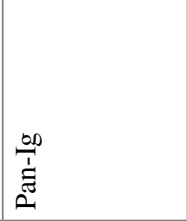 & ن & O. & 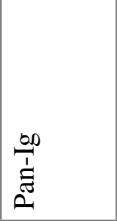 & 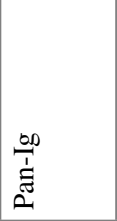 & U్ర & $\bigcup_{0}^{0}$ \\
\hline 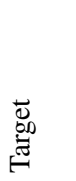 & $\frac{\mathscr{y}}{\tilde{n}}$ & $\frac{\mathscr{y}}{\frac{\tilde{n}}{2}}$ & $\frac{\mathscr{y}}{\tilde{n}}$ & $\frac{\mathscr{y}}{\frac{\tilde{n}}{n}}$ & $\frac{\mathscr{y}}{\frac{\vec{n}}{n}}$ & $\begin{array}{l}\frac{\mathscr{y}}{\tilde{2}} \\
\text { की }\end{array}$ & 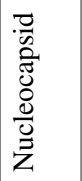 & 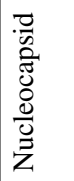 \\
\hline 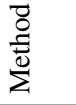 & $\begin{array}{l}\Xi \\
\Xi\end{array}$ & $\begin{array}{l}\Xi \\
\Xi\end{array}$ & 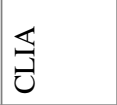 & $\begin{array}{l}\unlhd \\
己\end{array}$ & $\begin{array}{l}\Xi \\
\Xi\end{array}$ & 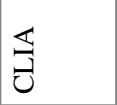 & $\underset{\sum}{\stackrel{\Xi}{J}}$ & $\underset{\sum}{\bigotimes}$ \\
\hline $\begin{array}{l}\frac{\bar{\Xi}}{0} \\
\frac{0}{0} \\
\frac{0}{0}\end{array}$ & 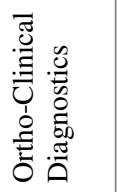 & 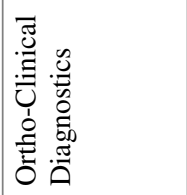 & 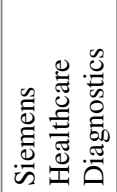 & 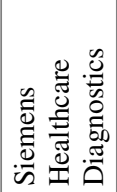 & 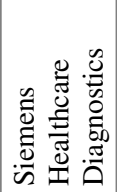 & 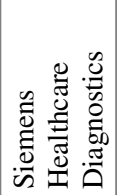 & 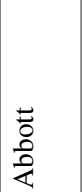 & $\begin{array}{l}= \\
0 \\
\frac{0}{2}\end{array}$ \\
\hline $\begin{array}{l}\vec{D} \\
\text { 定 }\end{array}$ & 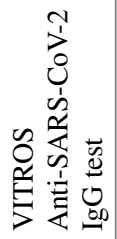 & 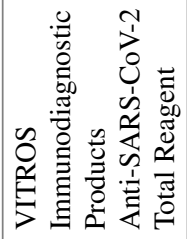 & 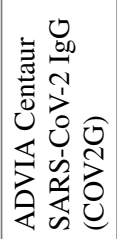 & 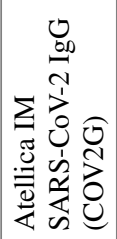 & 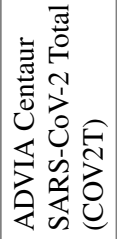 & 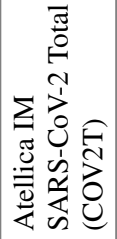 & 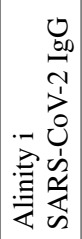 & 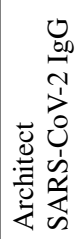 \\
\hline
\end{tabular}




\begin{tabular}{|c|c|c|c|c|c|c|c|c|c|}
\hline 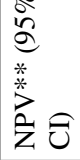 & $\begin{array}{c}\sigma \\
\hat{\alpha} \\
\circ \frac{1}{a} \\
\stackrel{a}{a}\end{array}$ & 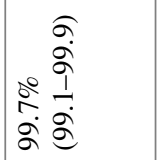 & 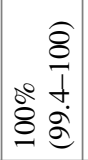 & 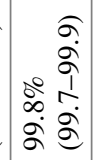 & 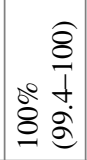 & 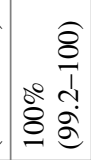 & 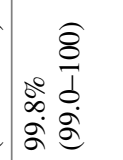 & 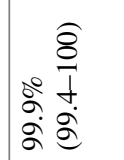 & 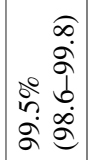 \\
\hline 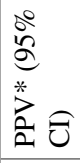 & 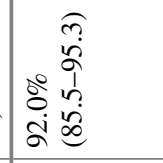 & 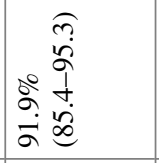 & 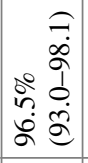 & 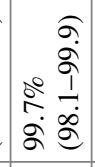 & 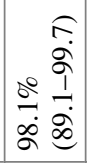 & 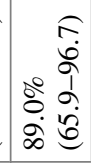 & 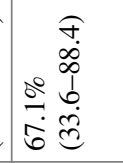 & 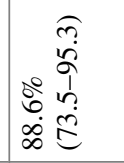 & 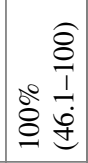 \\
\hline 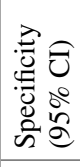 & $\begin{array}{r}\hat{a} \\
\hat{a} \\
0 \\
\dot{a} \\
\dot{a} \\
\dot{a}\end{array}$ & 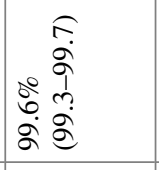 & 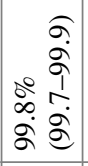 & 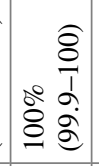 & 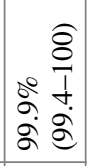 & 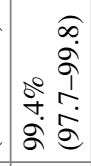 & 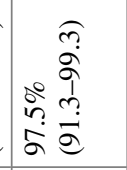 & 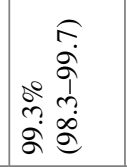 & 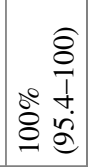 \\
\hline 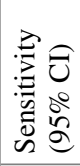 & 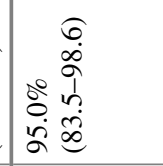 & 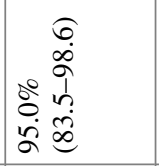 & 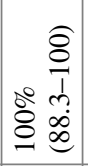 & 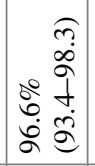 & 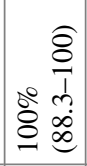 & 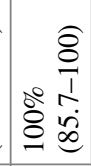 & 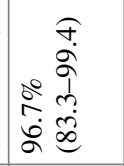 & 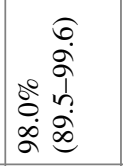 & 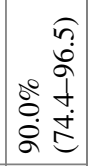 \\
\hline 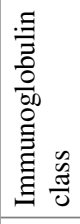 & $\sum_{\substack{a 0 \\
=}}$ & $\sum_{s=0}$ & 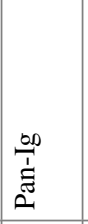 & 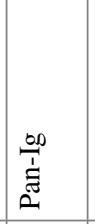 & 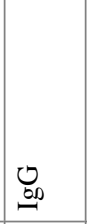 & $\sum_{\text {织 }}$ & 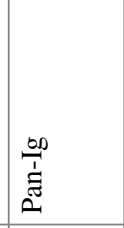 & 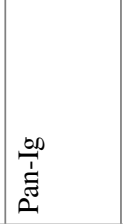 & $\begin{array}{l}0 \\
0 \\
-\infty\end{array}$ \\
\hline 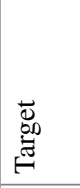 & $\begin{array}{l}\frac{0}{0} \\
\text { की }\end{array}$ & $\begin{array}{l}\frac{y}{2} \\
\frac{\pi}{n}\end{array}$ & 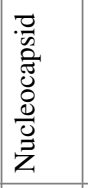 & $\frac{\mathscr{y}}{\overline{2}}$ & 总 & $\frac{y}{\vec{n}}$ & $\begin{array}{l}\frac{8}{0} \\
\frac{\vec{n}}{2}\end{array}$ & 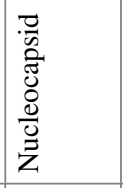 & 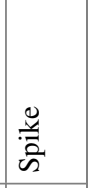 \\
\hline 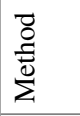 & 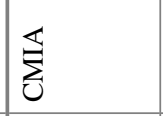 & 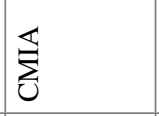 & $\begin{array}{l}\unlhd \\
己 \\
\text { J }\end{array}$ & $\begin{array}{l}\unlhd \\
己 \\
\text { J }\end{array}$ & 空 & 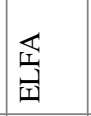 & 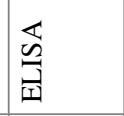 & 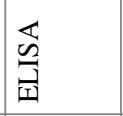 & 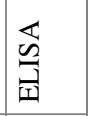 \\
\hline 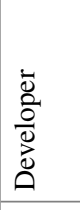 & 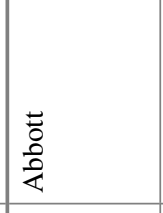 & 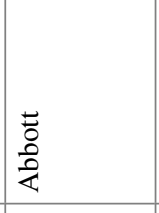 & 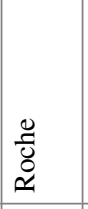 & 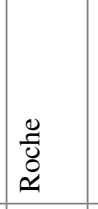 & 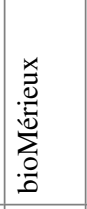 & 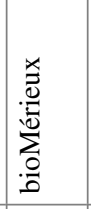 & 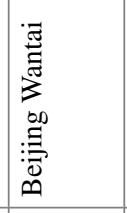 & 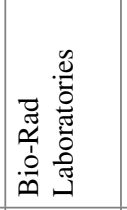 & 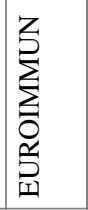 \\
\hline & 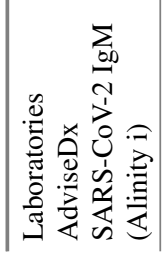 & 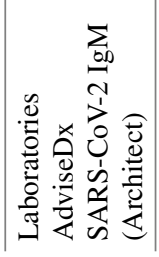 & 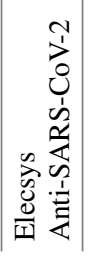 & 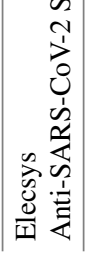 & 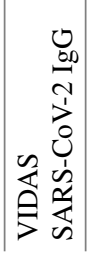 & 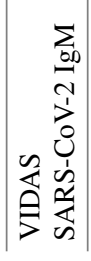 & 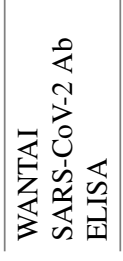 & 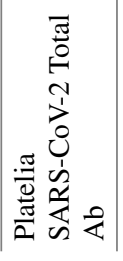 & 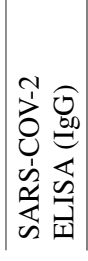 \\
\hline
\end{tabular}




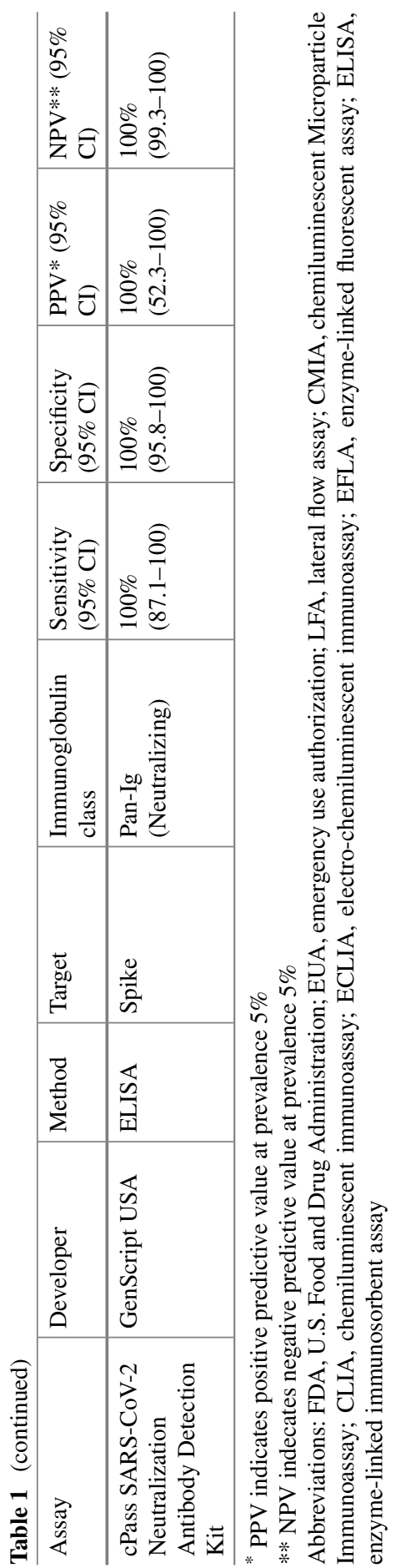


Table 2 Characteristics of antibody testing

\begin{tabular}{l|l|l|l|l}
\hline Test procedure & High-throughput & time-to-result & Sensitivity & Specificity \\
\hline Most simple & No & $10-30 \mathrm{~min}$ & Relatively low & High \\
\hline Complicate & Yes & $2-5 \mathrm{~h}$ & High & High \\
\hline Simple & Yes & $1-2 \mathrm{~h}$ & Highest & High \\
\hline Most complicate & No & $3-5$ days & - & -
\end{tabular}

Abbreviations: LFA, lateral flow assay; CLIA, chemiluminescent immunoassay; ELISA, enzymelinked immunosorbent assay

\subsection{Types of Antibody Testing}

Antibody tests for COVID-19 are markedly diverse in terms of test methods, target proteins, and immunoglobulin classes they detect (Table 1). Actually, multiple different types of assays are available to detect different aspects of the immune response and functionality of antibodies. Test methods vary widely, including: the lateral flow assay (LFA), which is often used for point-of-care testing, enzymelinked immunosorbent assay (ELISA), and other automated high-throughput assays, such as the electro-chemiluminescent immunoassay (ECLIA), chemiluminescent immunoassay (CLIA), and chemiluminescent microparticle immunoassay (CMIA). ${ }^{7}$ These assays mainly use spike protein, nucleocapsid protein, or their combination as target protein. The spike protein of SARS-CoV-2, which plays an important role in fusion and entry into the host cell, consists of an S1 domain that contains the receptorbinding domain (RBD) and S2 domain. The nucleocapsid protein of SARS-CoV-2 has a role in binding and packing of the viral RNA genome into a helical nucleocapsid structure during viral replication. These tests are divided into two categories to detect either binding (binding antibody detection) or neutralizing (neutralizing antibody detection) antibodies. The characteristics of each assay are summarized in Table $2 .^{8}$

\subsubsection{Binding Antibody Detection}

Lateral flow assays (LFAs) are widely used in human health for point-of-care testing (POCT) because of their convenience and ease of use. LFAs can be performed by a healthcare professional or by the patient in some instances, and in a range of settings, such as a hospital laboratory, clinic, or home. Sample preparation is very simple, and it only takes about 10-30 min to obtain the results. Typical LFAs use a test strip and require a drop of whole blood, plasma, or serum to detect the presence of immunoglobulins (for example, IgA, IgG, or IgM) against SARS-CoV-2 antigen.

\footnotetext{
${ }^{7}$ Ghaffari et al. (2020).

${ }^{8}$ Bastos et al. (2020).
} 
Although some LFAs need a specific instrument for each assay to measure the results, they are generally judged visually.

The ELISA assay, currently one of the most commonly used assays for antibody testing, is a laboratory-based test with an average time-to-result of 2-5 h. ELISA typically uses a plate whose surface of each well is coated with a specific antigen to bind to and detect the corresponding host immunoglobulins (for example, IgA, $\mathrm{IgG}$, or IgM) in whole blood, plasma, or serum samples. Then, the antigen-antibody complex is detected by a second antibody and a colorimetric or fluorescent substrate.

Other assays are usually performed using automated clinical laboratory equipment, and many samples are analyzed simultaneously. For example, the CLIA assay is similar to ELISA in that it takes advantage of high binding affinity between antigens and host antibodies but uses chemical probes that emit light through a chemical reaction to generate a positive signal. CLIA has a shorter average time-to-result $(1-2 \mathrm{~h}){ }^{9}$

CLIA and ELISA are both high-throughput laboratory-based assays with high concordance. $^{10}$

Surrogate virus neutralization tests (sVNT) are another type of binding antibody detection test. These assays are aimed to detect potential neutralizing antibodies that inhibit the interaction between RBD and angiotensin-converting enzyme 2 (ACE2, the cell receptor responsible for mediating infection by SARS-CoV-2).

These assays can be performed in BSL-2 laboratories.

\subsubsection{Neutralizing Antibody Detection}

The neutralizing antibody detection assay (neutralization assay) is a laboratory-based test that uses live virus and cell culture methods to determine if patient antibodies can prevent viral infection in vitro. The test procedures are laborious and must be performed in laboratories with designated biosafety certificates (for example, BSL-3) to culture SARS-CoV-2-infected cells and has a time-to-result of 3-5 days. ${ }^{11}$

\footnotetext{
${ }^{9}$ Ghaffari et al. (2020).

${ }^{10}$ Vashist (2020).

${ }^{11}$ Ghaffari et al. (2020).
} 


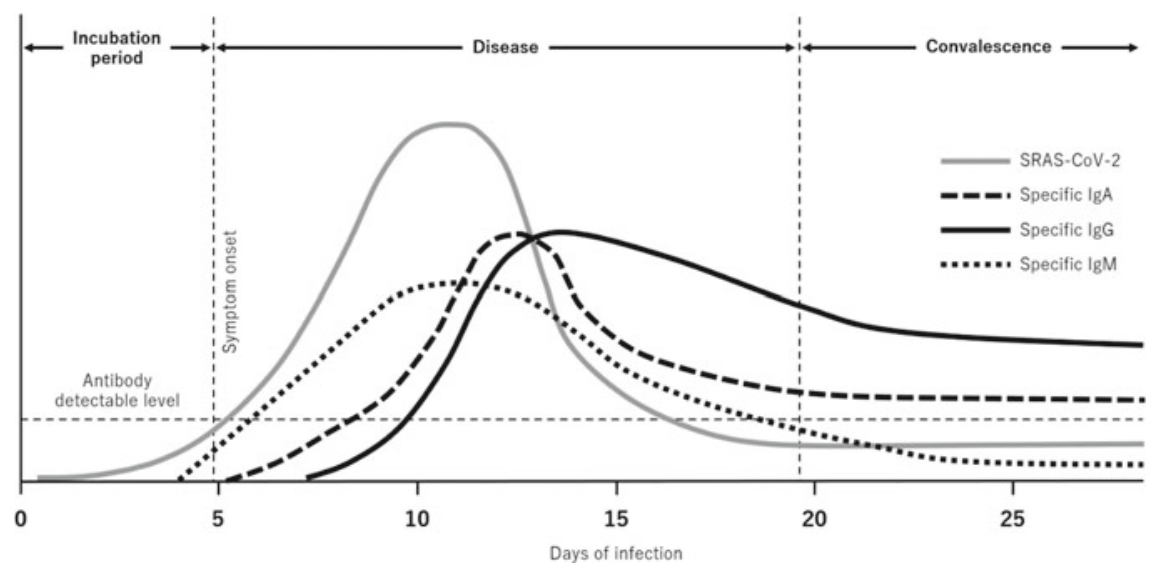

Fig. 1 Dynamics of seroconversion in COVID-19 patients ${ }^{12}$

\subsection{Dynamics of Seroconversion in COVID-19 Patients}

The timelines for the SARS-CoV-2 viral load and dynamics of IgA, IgG, and IgM are shown in Fig. $1 .{ }^{13}$ Antibodies are not detectable in the early phase of COVID-19 infection, but they can be detected after viral load increase.

Figure 2 shows the seropositive rate from symptom onset among COVID-19 patients. The results of two total antibody assays (A_Ab and C_Ab), two IgG assays (B_IgG and C_IgG), and two IgM assays (B_IgM and C_IgM) were evaluated, using 269 serum samples from 27 hospitalized patients at Kyoto University Hospital, Kyoto, Japan. The positive rate of antibody testing gradually rises until 15 days from symptom onset. The positive rate of pan-immunoglobulin assays is high and similar to that of $\mathrm{IgG}$ assays, and these are higher than that of IgM assays. As described in previous studies, ${ }^{14}$ the positive rate is relatively low in the early stage of COVID-19, because the host's immune response remains insufficiently established. Therefore, antibody testing may be less effective in this period. There are no clear data on how long the antibodies for SARS-CoV-2 are detectable. IgG could be detectable at least 49 days after symptom onset. ${ }^{15}$ On the other hand, IgM might not be detectable beyond 30 days after onset.

\footnotetext{
12 Wölfel et al. (2020).

${ }^{13}$ Ghaffari et al. (2020).

${ }^{14}$ Wölfel et al. (2020).

${ }^{15}$ Zhang et al. (2020).
} 


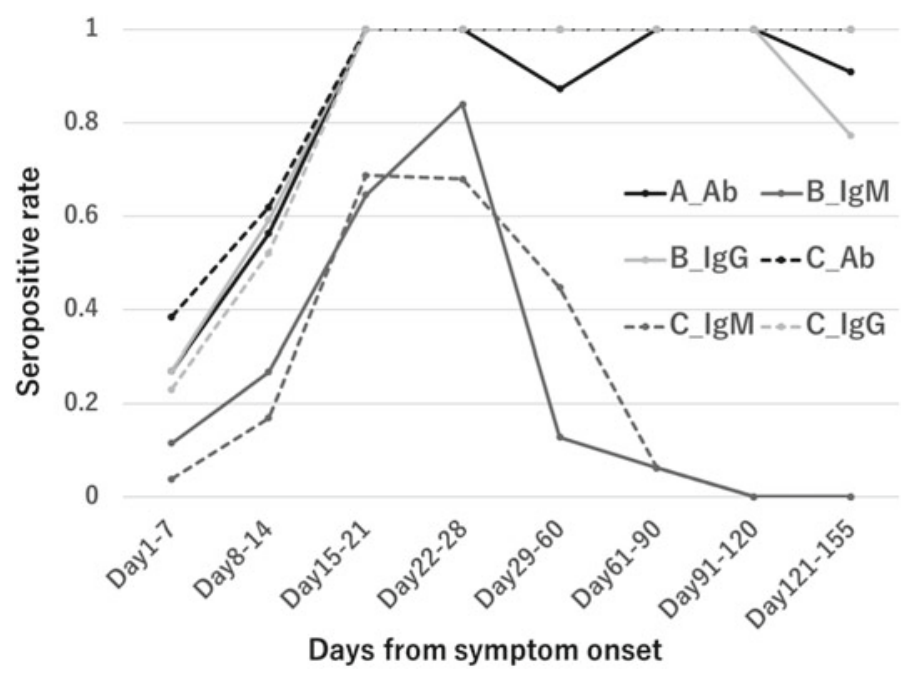

Fig. 2 Seropositive rate from symptom onset Pan-Ig assay: company A total antibody assay (A_Ab), company $\mathrm{C}$ total $\mathrm{Ab}$ assay (C_Ab) IgM assay: company B IgM assay (B_IgM), company C IgM assay (C_IgM) IgG assay: company B IgG assay (B_IgG), company C IgG assay (C_IgG)

\subsection{Antibody Test Selection}

To use antibody tests appropriately and effectively, it is important to comprehensively understand their performance characteristics and limitations. At present, antibody testing can be used most effectively for seroprevalence surveillance. The use of antibody tests to diagnose COVID-19 infection and evaluate vaccine immunity or convalescent serum is still controversial and requires further evaluation. When antibody tests are used in clinical settings, those with a high sensitivity of greater than 99.5\% should be chosen, especially in areas with a low prevalence of COVID-19.

Moreover, the tests should be repeated if necessary. The results of a single antibody test are not sufficiently accurate in low-prevalence populations. ${ }^{16}$ Thus, in these situations, a single test is rarely used to make a decision on whether a person has been infected previously or truly has specific antibodies to the virus. A second test that detects the presence of antibodies to a different viral protein would generally be needed to increase the accuracy of the overall test results. One concern regarding antibody testing should be noted: COVID-19 patients who are asymptomatic or with mild disease might have a lower immune response than patients with severe disease, and immunoglobulins could decline to undetectable levels throughout the course of infection. Therefore, well-designed research is needed, such as seroprevalence surveillance repeated every two or three months in the same study population.

\footnotetext{
${ }^{16}$ Interim Guidelines for COVID-19 Antibody Testing.
} 


\section{Epidemiological Study of COVID-19}

The novel coronavirus, SARS-CoV-2 infection (COVID-19), was first reported from China at the end of 2019. This virus has spread very rapidly, and the resulting pandemic remains an international public health emergency. Data on how many people have been infected comprise one of the most important epidemiological factors influencing public health policies. To date, many studies have been reported on national or local seroprevalence surveillance. During the present global pandemic of COVID-19, national surveillance data play a critical role in combatting and controlling the COVID-19 outbreak. In addition, local epidemiological data also play a key role, because local administrations should manage infection control in each outbreak area. Therefore, we perform seroprevalence surveillance in Kyoto city.

\subsection{Previous Study on Seroprevalence of COVID-19}

Seroprevalence surveillances of COVID-19 have been conducted all over the world, and several examples are presented as follows:

In the US, nationwide repeated cross-sectional seroprevalence research was conducted using a total of 177,919 serum samples from all 50 states between July and September 2020, ${ }^{17}$ although about 7.2 million people had been reported to be infected at the end of September 2020. Commercially available high-throughput IgG assays, all of which have high specificity, were used, including the Abbott ARCHITECT assay, Ortho-Clinical Diagnostics VITROS assay, and Roche Elecsys assay. At that time, the overall prevalence of COVID-19 in the US was less than $10 \%$, ranging from almost $0 \%$ (Alaska State) to over 20\% (New York State). The outbreak of COVID-19 remains an existential threat in the US. Confirmed cases of COVID-19 have doubled since October 2020. ${ }^{18}$ Therefore, a prevalence rate of $20 \%$ might be insufficient for herd immunity.

In the UK, seroprevalence was reported by Public Health England in October 2020. ${ }^{19}$ This research was conducted using the Euroimmun ELISA assay and healthy blood donors. London was the most prevalent area (about 15\%) in the UK, followed by the north west (10\%), and east of England (8\%).

In Germany, in early 2020, a seroprevalence of $0.91 \%$ was reported, using the Euroimmun ELISA assay. ${ }^{20}$

In Japan, the Ministry of Health, Labour and Welfare reported a COVID-19 seroprevalence of $0.038 \%$ in Tokyo, $0.02 \%$ in Osaka, and $0.004 \%$ in Miyagi Prefecture

\footnotetext{
17 Bajema et al. (2020).

${ }^{18}$ COVID-19 Dashboard by the Center for Systems Science and Engineering (CSSE) at Johns Hopkins University (JHU).

19 Sero-surveillance of COVID-19.

${ }^{20}$ Fischer et al. (2020).
} 


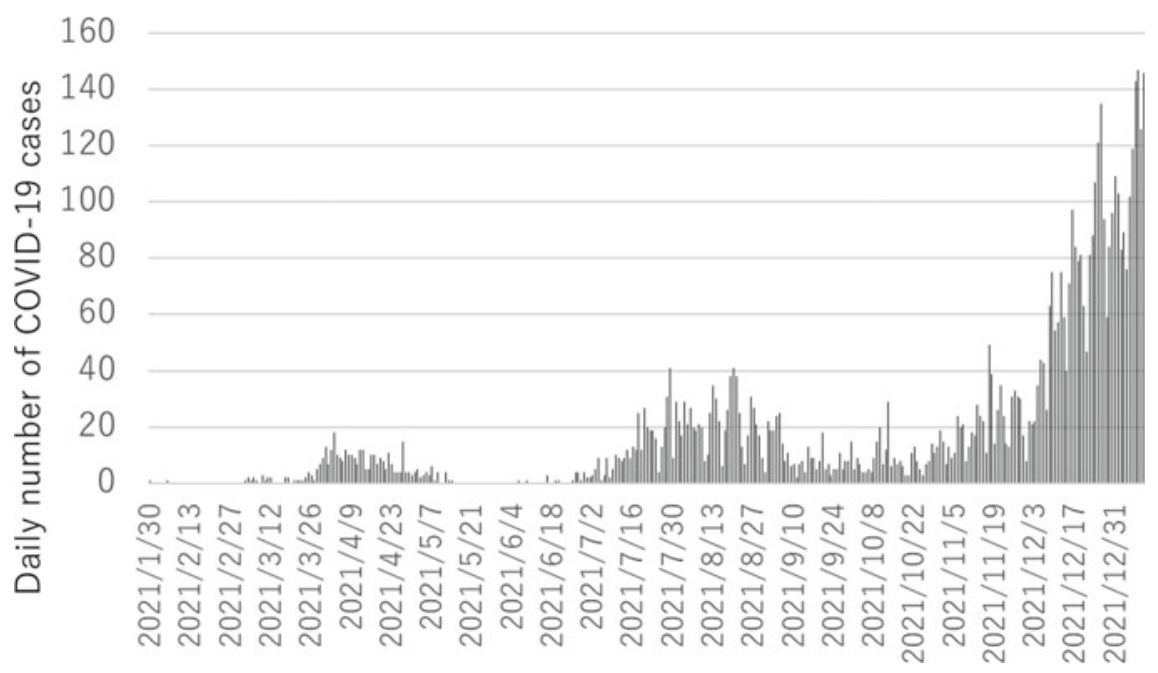

Fig. 3 Daily number of confirmed cases of COVID-19

in June 2020. The Abbott ARCHITECT assay and Roche Elecsys assay were used in this research. ${ }^{21}$

\subsection{Prevalence Surveillance in Kyoto City}

We started seroprevalence surveillance in Kyoto city from July 2020. The initial research was conducted between July and August 2020. Antibody testing was performed using the YHLO CLIA IgG and IgM kit, a high-throughput CLIA assay showing high-level concordance with the Roche Elecsys assay. In our research, the sensitivity and specificity of this IgG assay were $91.9 \%$ (95\% confidence interval: 87.7-95.1) and 100\% (95\%CI: 96.4-100), respectively. Among the 1737 serum samples from healthcare workers and essential workers analyzed, only eight $(0.46 \%)$ were seropositive for SARS-CoV-2.

As shown in Fig. 3, the daily number of confirmed cases of COVID-19 has been increasing since the end of October 2020.22 This local surveillance should be continued to evaluate and estimate the actual prevalence of COVID-19 in this region.

Only a year has passed since we first encountered COVID-19. Throughout that year, we have been trying to understand what "it" is and how "it" behaves. Due to concerted efforts, various testing modalities can now be used in clinical settings. At the present point, we have reached the stage where we need to determine how

\footnotetext{
${ }^{21}$ Result of Serosurveillance, in Japanese.

${ }^{22}$ COVID-19 in Kyoto Prefecture.
} 
to appropriately apply serology testing in order to control the COVID-19 pandemic through our case study in Kyoto city.

\section{References}

Bajema KL et al (2020) Estimated SARS-CoV-2 Seroprevalence in the US as of September 2020. JAMA Internal Medicine, 24 Nov 2020

Bastos ML et al (2020) Diagnostic accuracy of serological tests for covid-19: systematic review and meta-analysis. BMJ, 1 July 2020

Bohn MK et al (2020) IFCC Interim Guidelines on Serological Testing of Antibodies against SARS-CoV-2. Clin Chem Lab Med, 7 Oct 2020

COVID-19 Dashboard by the Center for Systems Science and Engineering (CSSE) at Johns Hopkins University (JHU). https://coronavirus.jhu.edu/map.html

EUA Authorized Serology Test Performance. www.fda.gov/medical-devices/coronavirus-disease2019-covid-19-emergency-use-authorizations-medical-devices/eua-authorized-serology-testperformance

Fischer B et al (2020) SARS-CoV-2 IgG seroprevalence in blood donors located in three different federal states, Germany, March to June 2020. Euro Surveill, 16 July 2020

Ghaffari A et al (2020) COVID-19 Serological Tests: How Well Do They Actually Perform?, 4 July 2020. https://www.ncbi.nlm.nih.gov/pmc/articles/PMC7400479/

Infectious Diseases Society of America Guidelines on the Diagnosis of COVID-19: Molecular Diagnostic Testing. www.idsociety.org/globalassets/idsa/practice-guidelines/covid-19/dia gnostics/idsa-covid-19-gl-dx-v2.0.0.pdf

Interim Guidelines for COVID-19 Antibody Testing. www.cdc.gov/coronavirus/2019-ncov/lab/res ources/antibody-tests-guidelines.html

Kyoto Prefecture. COVID-19 in Kyoto Prefecture, in Japanese. https://kyoto.stopcovid19.jp/

Ministry of Health, Labour and Welfare. Result of Serosurveillance, in Japanese. www.mhlw.go. jp/content/10906000/000640184.pdf

Public Health England. Sero-surveillance of COVID-19. www.gov.uk/government/publications/nat ional-covid-19-surveillance-reports/sero-surveillance-of-covid-19

Vashist SK (2020) In Vitro diagnostic assays for COVID-19: Recent advances and emerging trends. Diagnostics, April 5

Wölfel R et al. (2020) Virological assessment of hospitalized patients with COVID-2019. Nature, December 11

Zhang J et al. (2020) Serological detection of 2019-nCoV respond to the epidemic: A useful complement to nucleic acid testing. medRxiv (preprint), March 10

Masaki Yamamoto, MD, PhD is Senior Lecturer of the Department of Clinical Laboratory Medicine, Kyoto University Graduate School of Medicine and the Department of Clinical Laboratory and Department of Infection Control \& Prevention. He holds a Ph.D. in medicine from Kyoto University Graduate School of Medicine. His research interests have been antimicrobial resistance and molecular epidemiology of multi-drug resistant bacteria, and currently main interests are antibody testing and epidemiological study of COVID-19.

Yasufumi Matsumura, MD, PhD is an associate professor in Clinical Laboratory Medicine at the Kyoto University Graduate School of Medicine in Kyoto, Japan, and is Deputy Director of Clinical Laboratory and the Department of Infection Control and Prevention at the Kyoto University 
Hospital. He has been working on clinical and microbiological research on antimicrobial resistance in Enterobacterales, focusing on resistance mechanisms and molecular epidemiology. His current interests are molecular diagnosis and genomic analysis of SARS-CoV-2.

Miki Nagao, MD, PhD is a director and professor of infection control team and clinical laboratory department in Kyoto University Hospital. She also serves as a chief in COVID-19 research project organized by Kyoto University and Kyoto city. The principal areas of her research are infection prevention strategy for nosocomial pathogens, molecular epidemiology of multi-drug resistant pathogens and clinical laboratory medicine.

Open Access This chapter is licensed under the terms of the Creative Commons AttributionNonCommercial-NoDerivatives 4.0 International License (http://creativecommons.org/licenses/bync-nd/4.0/), which permits any noncommercial use, sharing, distribution and reproduction in any medium or format, as long as you give appropriate credit to the original author(s) and the source, provide a link to the Creative Commons license and indicate if you modified the licensed material. You do not have permission under this license to share adapted material derived from this chapter or parts of it.

The images or other third party material in this chapter are included in the chapter's Creative Commons license, unless indicated otherwise in a credit line to the material. If material is not included in the chapter's Creative Commons license and your intended use is not permitted by statutory regulation or exceeds the permitted use, you will need to obtain permission directly from the copyright holder.

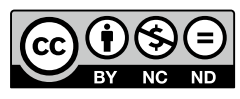

